\title{
TMS improves anxious aggressive behaviour in dogs: a case study
}

Dockx, Robrecht ${ }^{1}$

Peremans, Kathelijne';

Smolders, Ilse ${ }^{3}$;

Van Eeckhaut, Ann ${ }^{3}$;

Vlerick, Lise ${ }^{4}$;

Polis, Ingeborgh ${ }^{4}$;

Saunders, Jimmy $\mathrm{H}^{2}$;

Baeken, Chris ${ }^{1}$

${ }^{1}$ Department of Psychiatry and Medical Psychology, Ghent Experimental Psychiatry (GHEP) lab, Ghent University, Ghent, East Flanders, Belgium

${ }^{2}$ Department of Veterinary medical imaging and small animal orthopaedics, Faculty of

Veterinary Medicine, Ghent University, Merelbeke, East Flanders, Belgium.

${ }^{3}$ Department of Pharmaceutical Chemistry, Drug Analysis and Drug Information (FASC),

Research group Experimental Pharmacology, Center for Neurosciences (C4N), Vrije

Universiteit Brussel, Brussels, Belgium.

${ }^{4}$ Small Animal Department, Faculty of Veterinary Medicine, Ghent University, Merelbeke, East Flanders, Belgium.

Background: Accelerated high frequency repetitive transcranial magnetic stimulation (aHF-rTMS) has proven to produce fast clinical effects in humans suffering from psychiatric illnesses. Although dogs also frequently present behavioural symptoms similar to mental illness, nothing is known about the clinical influence in this species. A 5-year-old neutered male Belgian shepherd dog was presented with anxious aggressive behaviour.

Hypothesis: It was hypothesized that an aHF-rTMS treatment over the frontal cortex would improve the dogs' behaviour and alter its regional cerebral blood flow (rCBF) and monoamines.

Methodology: An aHF-rTMS protocol was applied twice (3 weeks separated) over the left frontal cortex ( 5 sessions, $20 \mathrm{~Hz}, 110 \% \mathrm{CMT}$ ). Each protocol was preceded and followed by a behaviour assessment and a $\left[{ }^{99 \mathrm{~m}} \mathrm{Tc}\right]$ HMPAO-SPECT scan. A Z-score for each VOI at each time point was obtained, a $|\mathrm{Z}|$-score $>3.09$ ( $P$-value of 0.001$)$ indicated significant differences. Cerebrospinal fluid (CSF) and serum was analysed for the detection of monoamines.

Results: An improvement of the dogs' aggressive behaviour was detected. At baseline, only a decreased rCBF of the left frontal cortex was noticeable (Z-score $=-3.87$ ). Twenty-four hours after the first protocol, the perfusion in the left frontal cortex was normalized but decreased in subcortical region $(Z$-score $=-6.97)$. Twenty-four hours after the second protocol, only the subcortical region was hypo-perfused $(Z$-score $=-6.88)$. Three weeks after each stimulation protocol, no deviations in the rCBF were found. Parallel changes (over time) of 3,4-Dihydroxyphenylacetic acid (DOPAC) concentrations in the serum and CSF were present (table 1).

Conclusion: This case study demonstrates that a single day aHF-rTMS treatment reduces a dogs' anxious/aggressive behaviour. This was accompanied by immediate and long- 
lasting alterations in the $\mathrm{rCBF}$ and DOPAC concentration. Thereby, this study confirms the interaction between the frontal cortex and the subcortical region in behaviour in dogs and puts DOPAC forward as possible biomarker.

\begin{tabular}{|c|c|c|c|c|c|}
\hline Monoamines & Baseline & $\begin{array}{c}1^{\text {st }} \text { protocol } \\
24 \text { hours }\end{array}$ & $\begin{array}{c}1^{\text {st }} \text { protocol } \\
3 \text { weeks }\end{array}$ & $\begin{array}{c}2^{\text {nd }} \text { protocol } \\
24 \text { hours }\end{array}$ & $\begin{array}{c}2^{\text {nd }} \text { protocol } \\
3 \text { weeks }\end{array}$ \\
\hline \multicolumn{6}{|l|}{ Serum } \\
\hline NAD & 3.62 & 4.55 & 4.16 & 4.01 & 6.23 \\
\hline $\mathrm{AD}$ & 11.50 & 8.81 & 14,20 & 10.31 & 26.96 \\
\hline DA & $\mathrm{Na}$ & $\mathrm{Na}$ & $\mathrm{Na}$ & $\mathrm{Na}$ & $\mathrm{Na}$ \\
\hline DOPAC & 26.39 & 122.41 & 33.067 & 92.76 & 27.90 \\
\hline HVA & 30.63 & 42.86 & 24.72 & 24.69 & 29.79 \\
\hline 5-HT & 21.59 & 18.62 & 29.97 & 25.23 & 25.17 \\
\hline 5-HIAA & $\mathrm{Na}$ & $\mathrm{Na}$ & $\mathrm{Na}$ & $\mathrm{Na}$ & $\mathrm{Na}$ \\
\hline \multicolumn{6}{|l|}{$C S F$} \\
\hline NAD & 1.45 & 1.63 & 1.54 & 1.23 & $\mathrm{Na}$ \\
\hline $\mathrm{AD}$ & 2.00 & 1.99 & 2.06 & 1.93 & $\mathrm{Na}$ \\
\hline DA & $\mathrm{Na}$ & $\mathrm{Na}$ & $\mathrm{Na}$ & $\mathrm{Na}$ & $\mathrm{Na}$ \\
\hline DOPAC & 0.16 & 0.49 & 0.25 & 0.87 & $\mathrm{Na}$ \\
\hline HVA & 12.57 & 19.40 & 12.70 & 11.31 & $\mathrm{Na}$ \\
\hline 5-HT & $\mathrm{Na}$ & $\mathrm{Na}$ & $\mathrm{Na}$ & $\mathrm{Na}$ & $\mathrm{Na}$ \\
\hline 5-HIAA & 2.20 & 3.70 & 2.35 & 2.49 & $\mathrm{Na}$ \\
\hline
\end{tabular}

Table 1: Concentrations ( $\mathrm{ng} / 100 \mu \mathrm{l})$ of 7 monoamines measured with HPLC. NAD = Norepinephrine; AD = Epinephrine; DOPAC = 3,4-Dihydroxyphenylacetic acid; HVA = Homovanillic acid; 5-HT = Serotonin; 5-HIAA = 5-Hydroxyindoleacetic acid; $\mathrm{Na}=$ missing data. 